American Journal of Applied Sciences 2 (8): 1228-1231, 2005

ISSN 1546-9239

(c) 2005 Science Publications

\title{
Demonstration of Optical Phase-conjugation in Methyl Green Dye-doped Thin Film
}

\author{
T. Geethakrishnan and P.K. Palanisamy \\ Center for Laser Technology, Department of Physics \\ Anna University, Chennai-600 025, India
}

\begin{abstract}
Optical Phase-Conjugation (OPC) has been observed in Methyl Green (MG) dye sensitized gelatin films in Degenerate Four-Wave Mixing (DFWM) configuration at $633 \mathrm{~nm}$ radiation from a He-Ne laser of total power $35 \mathrm{~mW}$. The mechanism of Phase-Conjugate (PC) wave generation associated with this dye-doped system was discussed. The dependence of the PC wave generation on the incident angle between the forward-pump and the probe beams and time of evolution were also studied. A maximum phase-conjugate beam reflectivity of about $0.13 \%$ has been observed in this dye-doped gelatin films.
\end{abstract}

Key words: Optical phase-conjugation, laser-induced gratings, organic dyes, four-wave mixing

\section{INTRODUCTION}

Over the last three decades, Optical PhaseConjugation (OPC) has been one of the major research subjects in the field of Nonlinear Optics (NLO) and quantum electronics. Optical phase-conjugation defines usually a special relationship between two coherent optical beams propagating in opposite directions with reversed wavefront and identical transverse amplitude distributions. The unique feature of a pair of PhaseConjugate (PC) beams is that the aberration influence imposed on the forward beam (signal) passed through an inhomogeneous or a disturbing medium can be automatically removed from the backward beam (phase-conjugated beam) passed through the same disturbing medium. Several technical approaches are there to efficiently produce the backward phaseconjugate beam. The first one is based on the Degenerate Four-Wave Mixing (DFWM) process, the second one is based on various backward stimulated scattering processes such as Brillouin, Raman, Raleighwing or Kerr and the third one is based on one-photon or multi-photon pumped backward stimulated emission (Lansing) process. Among these three techniques, the backward DFWM plays an important role in generating phase-conjugate beam, which is the formation of the induced holographic grating and the subsequent wavefront restoration via backward-pump (read) beam. OPC has a wide range of potential applications in the field of science and technology such as real-time holography, adaptive optics, and spectral filtering ${ }^{[1]}$. Organic dye-doped solid matrices have been very attractive materials in recent years for the generation of phase-conjugate waves ${ }^{[2-14]}$. Dyes having a strong absorption at the laser wavelengths and a long life time of their triplet state can generate the phase-conjugate wave at a low laser power. Organic dyes embedded in polymers have been also used as holographic recording media and permanent optical memories ${ }^{[15]}$.

In the present work, DFWM technique was used to generate the phase-conjugate waves of the continuouswave $\mathrm{He}-\mathrm{Ne}$ laser radiation of $35 \mathrm{~mW}$ at $633 \mathrm{~nm}$. An organic dye (Methyl Green - C. I.42590) -doped gelatin film was used as the Nonlinear Medium (NM) in the DFWM geometry.

\section{MATERIALS AND METHODS}

The organic dye used to sensitize the gelatin film is Methyl Green (MG) which belongs to the triphenylmethane ${ }^{[16]}$ groups. All the dyes of this series are derived from the hydrocarbon, triphenylmethane, and the tertiary alcohol, triphenylcarbinol, which are both colorless. The chromophore of this class is the quinonoid group. The chemical structure and molecular formula of this dye are shown in the Fig. 1. The UVvisible absorption spectrum of MG dye was studied using UV-2401 PC Spectrophotometer and it exhibits the peak absorbance at $631.5 \mathrm{~nm}$ as in Fig. 2. Gelatin films were prepared by removing silver halide from 10E75 Agfa Gaevert holographic plates by immersing them in sodium thiosulphate solution. The thicknesses of the films obtained were of the order of 10 microns. These plates were soaked in aqueous solutions of MG with appropriate dye concentrations for 2 minutes time duration and dried at room temperature. The optical quality of these dye-doped gelatin films obtained was very good. These films were used for this study without any further process. The Optical Density (OD) of the dye-doped gelatin film chosen for this work was approximately 1 .

Figure 3 shows the experimental configuration used to realize the optical phase-conjugation. In this work we used the standard DFWM geometry to generate the phase-conjugate wavefronts.

Corresponding Author: Prof.P.K.Palanisamy, Centre for Laser Technology, Department of Physics, Anna University, Chennai - 600 025, India, Tel: +91-44-22203160, Fax: +91-44-2220-3160 


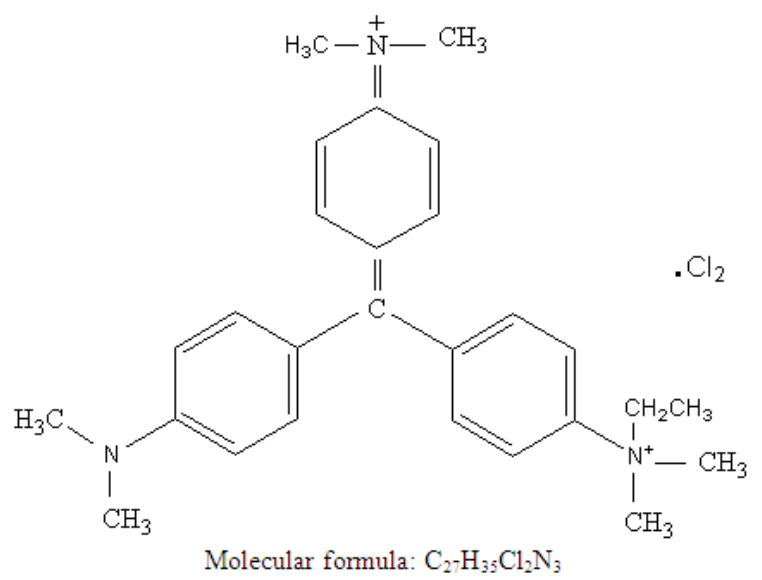

Fig. 1: Chemical structure of Methyl green dye (C. I = 42590)

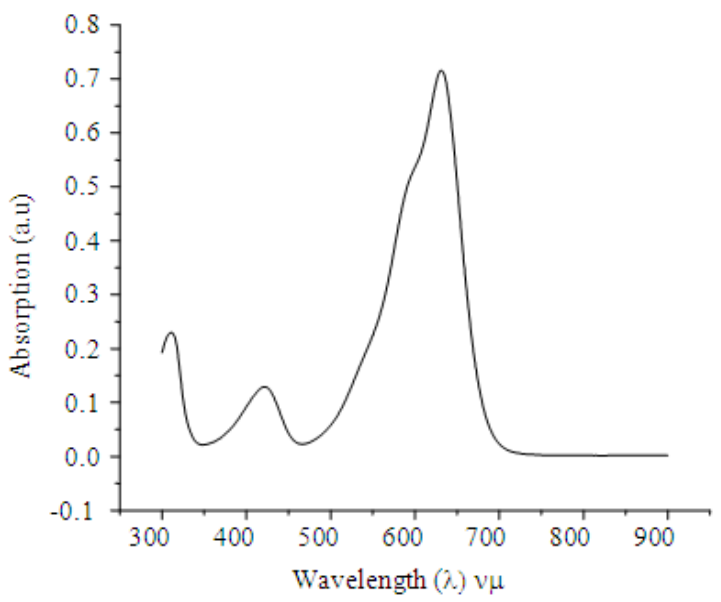

Fig. 2:The UV-VIS absorption spectra of Methyl green dye

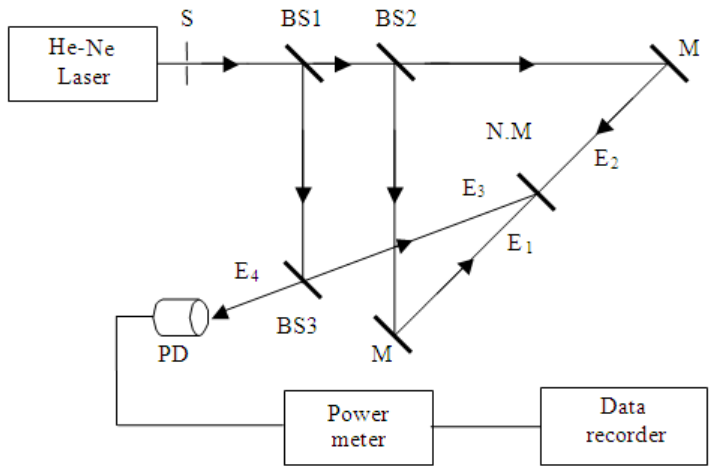

Fig. 3: Experimental set-up for the observation of PC wave, S-shutter, BS1, BS2, BS3 - beam splitters, M-mirror, N. M-nonlinear medium, PD-photo detector

A He-Ne laser (Coherent, 31-2140-000 - 35mW) beam at $633 \mathrm{~nm}$ was divided into three beams, two counter- propagating pump beams $E_{1}$ and $E_{2}$ namely forwardpump and backward-pump respectively and a probe beam $E_{3}$. The optical path lengths of all the three beams were made equal, so that they were coherent at the sample. The spot size of each of these three unfocused beams at the Nonlinear Medium (NM) was $1.25 \mathrm{~mm}$ in diameter. The constant intensity ratio of the probe beam $\left(E_{3}\right)$, forward-pump beam $\left(E_{1}\right)$ and backward-pump beam $\left(\mathrm{E}_{2}\right)$ used in this work was $\sim 1: 10: 10$. The angle of incidence $(\theta)$ between the probe beam and the forward-pump beam were varied between $5^{\circ}$ and $10^{\circ}$. The sample was exposed simultaneously to all these three beams. The phase-conjugated wave was separated from the probe beam using the beam-splitter BS3 and was detected with the help of a photo detector (Field Master ${ }^{\mathrm{TM}} \mathrm{GS}$ - Coherent Inc.). The experimental setup was mounted on a vibration isolation table (Melles Griot-Metric version) to avoid the destruction of the laser induced gratings formed in the MG dye-doped gelatin film due to mechanical disturbances.

\section{RESULTS AND DISCUSSION}

In MG dye sensitized gelatin film, the PC waves originate from two different processes. One of them is due to saturation of absorption while the other is due to the photo bleaching of the dye molecules at the excitation wavelength.

Absorption of a photon by MG results in transition of the $M G$ to the first excited singlet state $\left(S_{0} \rightarrow S_{1}\right)$. If the singlet-to-triplet crossover is considerable, the dye molecules will switch over to the triplet state $\left(S_{1} \rightarrow T_{1}\right)$, where they will remain for a relatively longer time as the triplet-to singlet transition is inhibited, and, consequently, these molecules will not be available for further absorption from the ground state. This will result in a saturation of absorption if the triplet lifetime is long enough. Thus, in the medium, the absorption becomes a function of intensity. Therefore, when the the two write beams interfere, the intensity pattern modulates the complex refractive index, which results in the formation of a grating. The fringe period $(\Lambda)$ can be determined by the well-known formula $\Lambda=\lambda / 2 \sin$ $(\theta / 2)$, where $\lambda$ is the laser wavelength, and $\pm \theta$ is the forward-pump and probe beam incident angles with respect to the normal to the nonlinear medium.

The second process to be considered is the photo bleaching of the dye molecules at the excitation wavelength. The existence of photo bleaching can be inferred from a simple experiment described as follows. MG dye-doped gelatin film was illuminated with 633 $\mathrm{nm}$ radiations at two different incident intensities and the corresponding transmittance of the sample was measured with respect to time. We observed that the transmittance of the sample increases from $33 \%$ to $40 \%$ and $33 \%$ to $45 \%$ in a time duration of about 30 minutes (Fig. 4) corresponding to the incident light intensities of $1.5 \mathrm{~W} / \mathrm{cm}^{2}$ and $3 \mathrm{~W} / \mathrm{cm}^{2}$ respectively. 


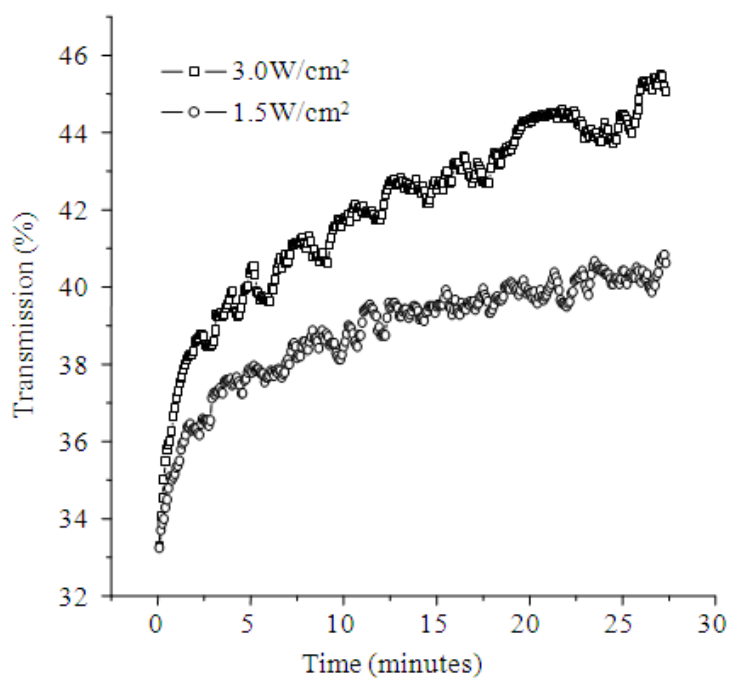

Fig. 4:Measured transmittance of the Methyl Green dye-doped gelatin film as a function of time

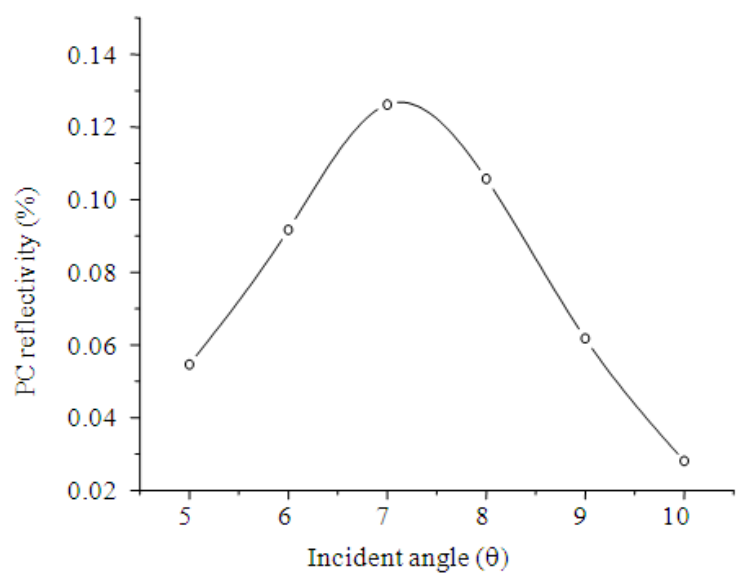

Fig. 5: Measured PC reflectivity as a function of incident angle between the probe and forwardpump beams

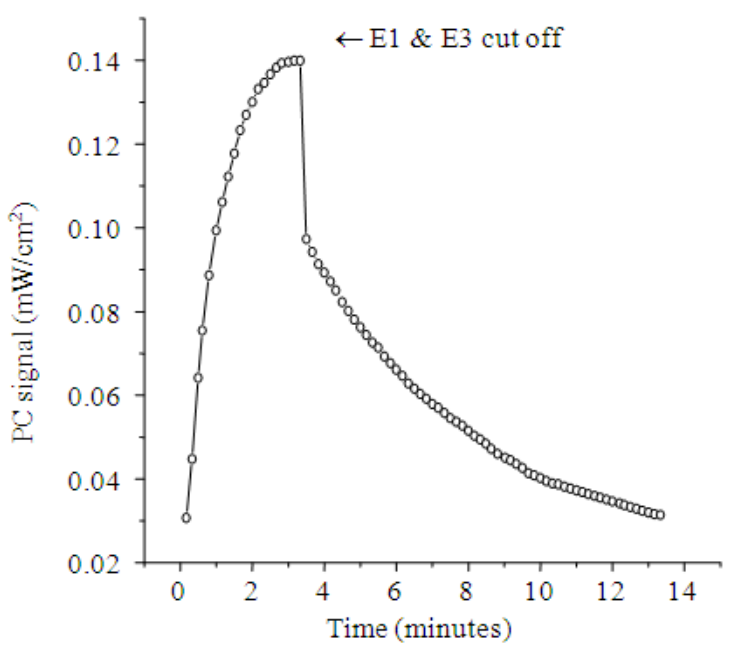

Fig. 6: Measured PC signal as a function of recording time
The observed increase of transmittance confirms the existence of light-induced bleaching process associated with this dye-doped system. For lower intensities the bleaching may be reversible but when higher intensities are used it may result in complete decomposition of dye molecules and hence become irreversible.

The phase-conjugate signal measurements were taken using the experimental setup shown in Fig. 3. To get maximum reflectivities it is necessary that the probe and pump beams should overlap exactly in the nonlinear medium. Figure 5 shows the PC reflectivities observed in the MG dye film as a function of recording angle $(\theta)$ between the forward-pump and the probe beams in the DFWM geometry. It is known that the phase-conjugated signal disappears when the angle between the beams is large enough ${ }^{[17]}$. This is because of less overlap between probe and pump beams in the dye film and small grating periods ${ }^{[18]}$. The highest phase-conjugate reflectivity $(0.13 \%)$ was observed at an angle of $7^{\circ}$.

Figure 6 shows the measured PC signal in the MG dye-doped gelatin film as a function of recording time. The initial rise to a peak within a few minutes is due to degenerate four-wave mixing and holographic processes; the sudden drop in the intensity of the PC signal after shutting off both the write beam $E_{1}$ and $E_{3}$ indicates the dominant contribution from DFWM processes. The presence of PC signal even after $E_{1}$ and $\mathrm{E}_{3}$ have been shut off is due to the holographic process, which decays rather slowly. A detailed explanation of these effects has been reported by Fujiwara and Nakagawa ${ }^{[4]}$.

\section{CONCLUSION}

We have observed low-intensity optical phaseconjugation (OPC) in Methyl green (MG) dye-doped gelatin films using degenerate four-wave mixing (DFWM) at $633 \mathrm{~nm}$ light radiating from a He-Ne laser. The maximum phase-conjugate beam reflectivity obtained from these dye films was about $0.13 \%$. The mechanism of PC wave generation associated with this dye-doped system was discussed. Dependence of PC wave generation on spatial separation between the forward-pump and the probe beams and time of evolution were studied. Since the MG dye-doped gelatin film was used at $633 \mathrm{~nm}$ and this may be suitable for low-power semiconductor lasers in the red wavelength region; MG dye-doped gelatin film may be a promising material for real-time double-exposure phase-conjugate interferometry applications.

\section{ACKNOWLEDGEMENT}

We thank All India Council of Technical Education (AICTE), Government of India, New Delhi for their generous support for this work. 


\section{REFERENCES}

1. Fisher, R.A., 1983. Optical Phase Conjugation. Academic, New York.

2. Prasad, B.R., R.K. Mohan, P.S. Narayanan, C.K. Subramanian and P. Chandrasekhar, 1988. Thermally induced optical phase conjugation by degenerate four-wave mixing. Curr. Sci. 57: 648-654.

3. Silberberg, Y. and I. Bar-Joseph, 1981. Low-power phase conjugation in thin film saturable absorbers. Opt. Commune., 39: 265-268.

4. Fujiwara, H. and K. Nakagawa, 1988. Phase conjugation in the fluorescein film by degenerate four-wave mixing and holographic process. Opt. Commune., 55: 386-390.

5. Moosad, K.P.B. and V.P.N. Nampoori, 1990. Saturable absorbers for low power phase conjugation. Pramana, 38: 281-287.

6. Divakara Rao, K. and K.K. Sharma, 1995. Dispersion of the induced optical nonlinearity in Rhodamine 6G doped boric acid glass. Opt. Commune., 119: 132-138.

7. Kumar, G.R., B.P. Singh and K.K. Sharma, 1989. Optical phase conjugation in Rhodamne 6G doped boric acid glass. Opt. Commune., 73: 81-84.

8. Mohajerani, E. and G.R. Mitchell, 1993. Temperature optimization of optical phase conjugation in dye doped polymer films. Opt. Commune., 97: 388-396.

9. Sreedhar, P.R. and R.S. Sirohi, 1993. Low power phase conjugation in phenosafranin dye films. Opt. Commune., 97: 83-87.
10. Kramer, M.A., W.R. Tompkin and R.W. Boyd, 1986. Nonlinear-optical interactions in fluorescent-doped boric acid glass. Phys. Rev. A, 34: 2026-2031.

11. Nakatsuka, H., D. Masuoka and T. Yamamoto, 1991. Optical phase conjugation in cooled organic dye film. Opt. Commun., 80: 215-218.

12. Miniewicz, A., S. Bartkiewicz, J. Sworakowski, J.A. Giacometti and M.M. Costa, 1998. With optical phase conjugation in polystyrene films containing the azobenzene dye Disperse Red 1. Pure Appl. Opt., 7: 709-721.

13. Haro-Poniatowski, E., M. Fernandez-Guasti, Camacho-Lopez and F. Ruiz, 1994. Phase conjugation and spatial grating formation in amorphous chalcogenide thin films. Physica A, 207: 329-333.

14. Tomov, I.V., T.E. Dutton, B. VanWonterghem and P.M. Rentzepis, 1991. Temperature dependence of degenerate four-wave mixing in azo dye doped polymer films. J. Appl. Phys., 70: 36-40.

15. Liu, Y., H. Wang, M. Tian and J. Lin, 1995. Multiple-hologram storage for thin layers of Methyl Orange dyes in polyvinyl alcohol matrices. Opt. Lett., 20: 1495-1497.

16. Venkataraman, K., 1971. The Chemistry of Synthetic Dyes. Academic press, New York.

17. Keinonen, T., P. Riihola, H. Huttu and S. Parkkonen, 1998. Dye films for optical demonstrations in the undergraduate laboratory. Opt. Materials, 11: 79-86.

18. Reddy, B.R., P. Venkateswarlu and M.C. George, 1991. Laser induced gratings in a styryl dye. Opt. Commune., 84: 334-338. 